\title{
VERBOS Y CONSTRUCCIONES CAUSATIVAS ANALÍTICAS EN PORTUGUÉS Y EN ESPAÑOL
}

\author{
Aucusto Soarfis da Silva \\ Universidade Católica Portuguesa - Braga \\ assilva@facfil.ucp.pt
}

\begin{abstract}
Resumen
Whon a causative meaning is expressed by an independent verbal element, we may identify the construction as analytic, periphrastic, or syntactic. Romance languages present greater granmatical elaboration of analytic causative constructions than other languages. Contradicting a long formalist (gencrative) tradition in general (and Romance) linguistics, the grammatical complexity of the causative constructions becomes explicable in the light of their semantic import and conceptual motivations. This paper offers a conceptual analysis of the analytic causative constructions in Portuguese and in Spanish. We will begin with a survey of the semantic properties of the causative verbs. Based on the Talmy's $(1988,2000)$ force dynamics model, four force-dynamic patterns are described, which are instantiated in fazer/hacer 'do/make', manter/mantener 'keep' and deixar/dejar 'leave/let'. Other analytic causative verbs such as mandar 'order', forçar/forzar 'force', obrigar/obligar 'oblige', and levar/levar 'lead', are considered. After that, we go on to analyze the grammar of the four main causative constructions in Portuguese and in Spanish, namely infinitival, finite, reflexive and resultative constructions. The conceptual factors (or imagery, Langacker, 1987, 1991) of the three infinitival complement constructions of Portuguese arc bricfly analyzed, which represent a more elaborate continuum of event integration and of causee autonomy and causcr control. Finally, we briefly describe the compatibilities between the verbs and constructions, and include a reference to the alternative construction with the finite complement, and to the causativc-rcflexivc and causative-resultative constructions.
\end{abstract}

\section{INTRODUCCIÓN*}

De entre las varias formas lingüísticas de expresión de la causación, la más regular y productiva en diferentes lenguas es la analitica o perifrástica (Shibatani y Pardeshi, 2002:109), denominada también la de los causativos sintácticos, es decir, estructuras de dos verbos (típicamente) donde uno expresa el predicado causal (evento causante) y el otro el predicado de efecto (evento causado). En la causación analítica, el predicado causal (fazer/ hacer o deixar/dejar, por ejemplo) tan sólo expresa la noción de causa (u otras nociones íntimamente relacionadas, como manipulación, posibilidad, permiso), sin ningún contenido léxico más específico, lo que hace que esté conceptualmente dependiente del predicado de efecto (Kemmer y Verhagen, 1994:117).

Este texto es cl fundamento de los cursos impartidos en la Facultad de Filosofía y Letras, de la Universidad de Alicante, sobre esta temática, en marzo de 2002 y 2003 . Mi agradecimiento a todos los presentes por sus interesanles sugerencias señaladas y comentadas. 
La causación analítica exhibe una gran complejidad gramatical (mayor en las lenguas románicas que en las lenguas germánicas), que se puede explicar en la base de factores, ya no tanto formales - como nos ha habituado la tradición gencrativista-, sino conceptuales y semánticos. En el marco de la Lingüística Cognitiva, y en la secuencia de otros estudios (Soarcs da Silva, 1999, 2000, 2003a, b, 2004, en prensa 1, 2, 3), analizaremos en este estudio la causación analítica en portugués y en español, con mayor incidencia en portugués. Comenzaremos por los verbos causativos $\mathrm{y}$, apoyándonos en el modelo de dinámica de fuerzas de Talmy $(1988,2000)$, describiremos cuatro esquemas de causación, realizados en fazer/hacer, manter/mantener y deixaridejar, y además tendremos en cuenta otros verbos causativos. Pasaremos, a continuación, a las construcciones causativas, más complejas en portugués que en las otras lenguas románicas, entendiendo construcciones como pares de forma y significado que existen independientemente de verbos particulares (Goldberg, 1995:1). En el marco de la Gramática Cognitiva de Langacker (1987, 1991, 1999), analizaremos las tres construcciones infinitivas del portugués, las que envuelven un contimutm de integración de los eventos causante y causado y de autonomía de lo causado y control de lo causante. Haremos también una descripción bastante sumaria de las compatibilidades entre verbos y construcciones y breves referencias a las construcciones causativas de complemento finito, causativo-reflexiva y causativo-resultativa.

\section{Verbos Caljsativos}

En un estudio seminal en semántica cognitiva, Talmy (1988) -ligeramente modificado en Talmy (2000)- desarrolló una teoría sobre un sistema cognitivo fundamental al que denominó dinúmica de fuerzas y la propuso como «a gencralization over the traditional linguistic notion of 'causative'» (Talmy, 2000:409). En su forma más básica, este sistema envuelve una oposición entre dos entidades, una que cjerce fucrza (la entidad focal) y otra que ejerce una contra-fuerza, a las que Talmy llama Agonista y Antagonista, respectivamente (y que se corresponden con lo que tradicionalmente se designa por causado y causante, respectivamente). Talmy elabora un sistema de padrones de dinámica de fuerzas basado en cuatro dimensiones:

(i) tendencia intrínseca de fuerza de lo Agonista hacia el movimiento o acción vs. hacia el reposo o inacción;

(ii) cambio en el tiempo vs. no-cambio; con otras palabras, oposición de fuerzas mutable (inicio/cese de la influencia) vs. oposición de fuerzas estable (continuación/noocurrencia de la influencia);

(iii) influencia ('causar') vs. no-influencia ('dejar'): el estado resultante de lo Agonista es el opuesto del de su tendencia intrínseca en 'causar', mientras que es el mismo en 'deixar';

(iv) entidad más fuerte: Antagonista vs. Agonista.

Utilizando las convenciones simbólicas y los diagramas de Talmy, la Figura 1 sistematiza cuatro padrones de dinámica de fuerzas con Antagonista como fuerza más fuerte (ocho padrones, si incluimos la distinción aplicada a la tendencia de lo Agonista entre "hacia el 
movimiento' y 'hacia el reposo')'. Se indican los verbos causativos que realizan estos padrones.

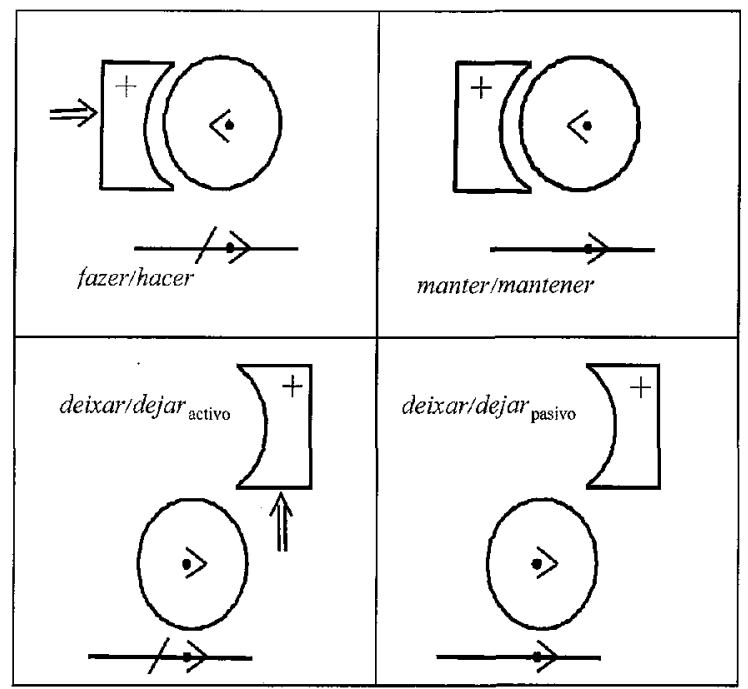

Figura 1. Padrones de dinámica de fuerzas

Los verbos fazer/hacer y manter/mantener y sus equivalentes en otras lenguas (como make y keep en inglés) realizan los dos padrones de 'causar', respectivamente: lo mutable (el padrón prototípico) y lo estable, mientras que los dos padrones de 'dejar', lo mutable (considerado por Talmy como protolípico) y lo estable, son realizados por el verbo deixar/ dejar y sus equivalentes, como el inglés let, en los sentidos de 'cesar de impedir, soltar' ('dejar' activo) y 'no impedir' ('dejar' pasivo), respectivamente. Todos estos padrones de dinámica de fuerzas se fundamentan en el dominio de la fuerza física, pero se proyectan cn los dominios psicológico, social, moral y mental.

La diferencia fundamental entre los causativos fazer/hacer y manter/mantener, por un lado, y deixar/dejar, por otro, se aclara en este marco de dinámica de fuerzas. Con fazer hacer y manter/mantener, lo Antagonista aplica una fuerza (física o no-física) contra la tendencia intrínseca de lo Agonista, mientras que con deixar/dejar lo Antagonista se abstiene o deja de ejercer una fuerza que pueda oponerse a la tendencia de lo Agonista. Consecuentemente, el resultado de la interacción de fuerzas para lo Agonista es lo opuesto al de su tendencia intrínseca en fazer/hacer y manter/mantener (es decir, lo Agonista sufre determi-

1 El círculo representa lo Agonista y la figura cóncava lo Antagonista; + : la entidad más fuerte; $>/ \bullet:$ tendencia intrínseca hacia el movimiento/reposo; $\longrightarrow$ : el resultado de la interacción de fuerzas es el movimiento; $\longrightarrow$ - el resultado de la interacción de fuerzas es el reposo. Los padrones que envuelven cambio en cl tiempo se indican a través de una flecha junto a lo Antagonista, de inicio o cese de influencia, $y$ de un trazo en la línea del estado resultante. 
nado cambio), sin embargo es el mismo que en deixar/dejar. Comparemos los ejemplos (1) y (2). María, el libro y el agua tienden, en (1), a permanecer en el lugar o en el estado donde se encontraban, pero su estado resultante es el movimiento o el cambio, debido a la acción de lo Antagonista, que actúa como motor. Por cl contrario, en (2) tienden al movimiento o cambio de estado y así continúan, ya que lo Antagonista no actúa como barrera².

(1) a. O João fez a Maria sair. Juan hizo salir a María.

b. O João fez cair o livro. Juan hizo caer el libro.

c. A doença faz reflectir a Maria.

La enfermedad hace reflexionar a Maria.

d. O vento fez correr a água.

El viento hizo correr el agua.

(2) a. O.João deixou a Maria sair.

Juan dejó salir a María.

b. O João deixou cair o livro.

Juan dejó caer el libro.

c. A doença deixa a Maria reflectir:

La enfermedad deja reflexionar a Moria.

d. A tampa deixa passar a água.

La tapa deja pasar el agua.

Comparemos ahora manter/mantener y deixar/dejar (en el sentido pasivo de "no intervenir'). Como se puede comprobar en los ejemplos (3) y (4), en ambos la oposición de fuerzas es estable: lo Antagonista no toma ni deja su condición de fuerza oponente sino que continúa en esa condición - su fuerza es continuamente ejercida en (3) y continuamente no ejercida en (4). En ambos, el resultado de la oposición de fuerzas es el mismo: la no alteración del estado de lo Agonista, ya sea cl movimiento o el reposo. La diferencia entre los verbos causativos es la misma que ya hemos comprobado antes. El estado resultante de la interacción es diferente de la tendencia de lo Agonista, porque lo Antagonista de manter/mantener ejerce una fuerza contra la tendencia de lo Agonista. En (3a, b), lo Agonista ticnde al movimiento (el tesoro tiende a dejar el lugar donde se encontraba y María tiene tendencia hacia la inquietud), no obstante el estado resultante es el reposo (cl tesoro permanece en su lugar y María permanece tranquila) ya que lo Antagonista actúa como barrera; y en (3c) lo Agonista tiende al reposo (los niños tienden a parar de jugar), sin embargo, el estado resultante es el movimiento (los niños permanecen jugando) porque lo Antagonista ahora actúa como motor. Por el contrario, en deixar/dejar el estado resultante es igual al de la tendencia de lo Agonista: en ( $4 a, b)$ el tesoro y María tienden al reposo y así continúan, y en $(4 \mathrm{c})$ los niños tienden al movimiento y también continúan así no obstante lo Antagonista está presente y no actúa ni como motor ni como barrera. Se puede concluir de este modo que 'dejar las cosas como están' y 'mantener las cosas como están' expresan construcciones

2 Nólese que lo Agonista de la construcción con fazer/hacer puede tender al movimiento y, en ese caso, lo Antagonista actúa como barrera (cfr. O policia fez parar o automobilista / El policia hizo parar al condluctor), así como lo Agonista de la construcción con dejar puede tender al reposo y, en ese caso, lo Antagonista no actúa como motor (cfr. 4b, a continuación). 
conceptuales radicalmente diferentes de la misma situación del mundo real (para un análisis detallado, véase Soares da Silva, en prensa 2).

(3) a. O João manteve'guardou o tesouro na gruta. Juan mantuvo/guardó el tesoro en la gruta.

b. O João manteve a Maria calma. Juan mantuvo a Maria tranquila.

c. O João manteve as crianças a brincar. Juan mantuvo a los niños jugando.

(4) a. O João deixou o tesouro na gruta. Juan dejó el tesoro en la gruta.

b. O João deixou a Maria em paz. Juan dejó a María en paz.

c. O João deixou as crianças brincarem. Juan dejó a los niños jugar.

Fazer/hacer y manter/mantener perfilan, entonces, una causación más directa, mientras que deixar/dejar perfila una causación más indirecta, o antes una causación negativa, cn el sentido de que lo Antagonista no ejerce una fuerza que pueda impedir, o ejerce una fuerza en orden a cesar de impedir, la disposición natural de lo Agonista - es precisamente por causa de esta no interferencia, de este acto negativo que lo Agonista se «deja libre» para seguir su curso natural ${ }^{3}$.

Naturalmente que existen otros verbos causativo analíticos, pero son elaboraciones o extensiones de éstos. Adcmás, cada uno de estos causativos es un concepto bastante complcjo. El análisis de su complejidad semántica sobrepasa ampliamente los límites del presente estudio. Dejaremos aquí tan sólo algunos elementos de sistematización.

Fazer/hacer funcionan como verbos de creación, verbos de causación (física) y verbos de manipulación interpersonal. Tanto fazer como hacer presentan un campo más amplio de aplicación semántica que, por ejemplo, el equivalente del francés faire y del inglés make. Los causativos fazer y hacer pueden expresar diferentes grados de fuerza coerciva, como 'forzar', 'obligar', 'hacer' y además un sentido no intencional de 'causar'. El ejemplo (5) puede interpretarse bien en un sentido volitivo, más o menos coercivo, bien en un sentido de causación involuntaria (siendo, en cste último caso, perfectamente aceptable introducir el sintagma sem querer/sin querer).

\section{A Marie fê-lo voltar a casa.}

María lo hizo volver a casa.

Campo de uso aún más amplio tiene cl causativo del italiano fare, desde la causación más coerciva a la más inductiva y llegando incluso a algunos usos de 'dcjar'. Por su parte, el causativo del francés faire difiere de sus cognatos románicos por poseer una escala de fuerza menor. Como veremos más adelante, el verbo portugués fazer es aquél que tiene un campo más amplio de construcciones gramaticales causativas.

3 Para un análisis más detallado de las propiedades tanto de lo causante como de lo causado de fazer/deixar y hacer/dejar, véase Rernárdez (2001) y Soares da Silva (2004, en prensa 3). 
Por otro lado, el inglés presenta una variación onomasiológica del concepto de 'causar' que el portugués, el español y las otras lenguas románicas no poseen. Esta variación incluye, además de make, los verbos have, get y cause y todos estos verbos causativos son semánticamente complejos.

El verbo íbérico deixar/dejar -así como los restantes cognatos románicos (francés laisser, italiano lasciare, rumano a lăsa) - exhibe un vasto y diversificado conjunto de sentidos, distribuidos por dos grupos principales, equivalentes a los verbos del inglés leave y let (Soares da Silva, 1999, 2003a, b). Cuando aparece construido con complemento verbal, deixar/dejar posee tres sentidos principales, concretamente 'no impedir', 'cesar de impedir, soltar' y 'permitir', respectivamente cjemplificados en (6)-(8). El primer sentido expresa una actitud 'pasiva' del sujeto principal, mientras que los otros dos expresan una actitud 'activa'.

(6) O João pôs-se a fazer disparates, e eu deixei-o fazer.

Juan se puso a hacer tonterias, y se las dejé hacer.

(7) O João deixou o pássaro vaar (abrindo a gaiola).

Juan dejó volar al pájaro (abriendo la jaula).

(8) O João pediu-me para ir ao cinema, e eu deixei-o ir:

Juan me pidió ir al cine, $y$ lo dejé ir.

El sentido de 'no intervenir, no impcdir' (6), particularmente cuando envuelve un agente, es el sentido prototípico de deixar/dejar con complemento verbal y de sus equivalentes románicos. El sentido de 'cesar de impedir, soltar' (7) es cl prototipo diacrónico del verbo románico. El tercer sentido (8) envuelve claramente la noción normativa de concesión de permiso. Como hemos visto, el primer sentido envuelve una dinámica de fuerzas de no ocurrencia de interferencia (ausencia de barrera), mientras que el segundo consiste en un cese de interferencia (eliminación de barrera).

Como ya hemos comprobado, el significado causativo se mantiene en otra construcción donde lo causado o Agonista se concibe, no dinámicamente, como en los ejemplos (6)-(8), sino estáticamentc, como en $(4 a, b)$. Al contrario del inglés, en las lenguas románicas el verbo es el mismo; tan sólo difiere la construcción sintáctica de su complemento: complemento verbal, prototípicamente complemento de infinitivo, cuando lo Agonista tiende al movimiento y complemento nominal cuando lo Agonista tiende al reposo.

Existe en portugués además otro causativo analítico con un comportamiento sintáctico idéntico al de fazer y dejxar - es el verbo mandar como causativo de orden 'ordenar', djferente de mandar como causativo de movimiento 'enviar'. El causativo de orden mandar cxpresa una configuración de dinámica de fuerzas idéntica a la de fazer: ambos perfilan una fuerte influencia ejercida por lo causante sobre lo causado. Difieren en el resultado o «parámetro del éxito» (Jackendoff, 1990:132): fazer, por lo menos en sentido coercivo, tiene un resultado alcanzado, mientras que el resultado de mandar es indeterminado, como se ilustra en los ejemplos (9)-(10)4.

4 Como verbo de orden, mandar en español tolera la construcción con infinitivo directo, cuando el inlinitivo es intransitivo, como en lo mandé veni; lo mandé ir a tu casa (aungue csta construcción sea poco habitual), sin embargo ya no con infinitivo transitivo (??le mandé traer un libro) (Cano Aguilar, 1981:351). Diferente es el caso de mandar como verbo de movimiento causado en la construcción con infinitivo preposicionado, como en Antonio mandó a Maria a buscar los papeles. 
(9) Fi-lo ir embora, ?" mas ele não foi.

'Lo hice marchar, ?* pero él no se fue.'

(10) Mandei-o ir embora, mas ele não foi.

'Lo mande marchar, pero el no se fue.'

Es decir, mandar es un verbo no implicativo. $\mathrm{Y}$, como verbo directivo de orden, envuelve una interacción necesariamente interpersonal y, por eso, les impone restricciones de intencionalidad tanto al sujeto principal como al sujeto subordinado.

Hay otros verbos causativos analíticos que expresan ya sea causación coerciva interpersonal (o necesidad), en grado superior al de fazer/hacer de coerción, como forçariforzar u obrigar/obligar, ya sea una causación inductiva interpersonal, como conduzir/conducir, induzir/inducir, levar/levar, persuadir, convencer. Estos verbos constituyen una categoría sintáctica distinta: todos toman un complemento infinitivo con preposición c, igualmente al contrario de los anteriores, generalmente no se gramaticalizaron para la expresión de la causación (como excepción tenemos levar/llevar).

\section{VARIEDAD DE CONSTRUCCIONES CAUSATIVAS}

El portugućs, cl español (y otras lenguas románicas) poseen cuatro construcciones causativas analíticas principales, identificadas y ejemplificadas en (11)-(14). Es la lengua portuguesa la que presenta una mayor complejidad en la construcción con complemento infinitivo, patente en (11).

(i) construcción causativa con complemento infinitivo

(11) a. A Maria faz/mandu/deixa as crianças sairem.

Maria - hace/manda/deja - los niños - salir-INI ILLEX

b. A Maria faz/manda/deixa as crianças sair. Maria hace/manda/deja a los niños salir.

c. A Maria faz/manda/deixa sair as crianças. Maria hacetmanda/deja salir a los niños.

(12) a. A Maria forçalobriga/leva as crianças a sairem. María - fucrza/obliga/lleva - los niños - a salir-INF FLEX

b. A Maria força/obrigalleva as crianças a sair: Maria fuerzalobligalleva a los niños a salir.

(ii) construcción causativa con complemento finito

(13) a. A Maria faz com quémanda/deixa que as crianças scium. Maria hace/mandaldeja que los niños salgan.

b. A Maria forçalobrigalleva a que as crianças saiam. Maria fuerzáobligafleva a que los niños salgan.

(iii) construcción causativo-reflexiva

(14) a. A Maria deixou-se cair no sofá. Maria se dejó caer en el sofá.

b. A Maria fez-seldeixou-se levar pelo amigo. Maria se hizo/se dejó llevar por el amigo.

c. A Maria obriga-se (a si mesma) a fazer o exercicio. Maria se obliga (a si misma) a hacer el ejercicio. 
(iv) construcción causativa predicativa (o causativo-resultativa)

(15) A Maria/lsso Jez-me/pôs-me/tornou-me/deixou-me feliz. Maria/Eso me hizo/me puso/me volviólme dejó feliz.

Vamos a analizar, a continuación, los significados de estas construcciones y los factores conceptuales de su elección, con incidencia mayor en las construcciones con complemento infinitivo y con complemento finito.

Comparativamente, las lenguas románicas, y especialmente el portugués, se caracterizan por una mayor elaboración gramatical de la integración de los eventos causante y causado (particularmente, estructuración del evento causado), que, por ejemplo, el inglés y otras lenguas germánicas. Dicho con otras palabras, la rclación entre causa y efecto - tomados ambos como eventos - tiene un grado más elevado de destacabilidad perspectival (o atención a diferentes partes del episodio causativo) y de destacabilidad estructural (o distintividad) en las lenguas románicas, en particular en el portugués, que en las lenguas germánicas.

\section{TRES CONSTRUCCIONES CAUSATIVAS CON COMPLEMENTO INFINTIVO EN PORTUGUÉS: TRFS MÁGENFS} SOBRE UNA MISMA BASE

Existen en portugués, no sólo dos, como en español y en otras lenguas románicas, sino tres construcciones de complemento infinitivo donde parlicipan los verbos causativos fazer 'hacer', mandar 'mandar' y deixar 'dejar' y además los verbos perceptivos's ver 'ver' y ouvir 'oír'. Estas construcciones se presentan en (a), (b) e (c) de (16)-(19).

(16) a. A Maria fez/mandou/deixou os miúdos correrem.

Maria - hizo/mando/dejó - los niños - correr-INF FLEX

b. A Maria feztmandou/deixou os miuidos correr.

María - hizo/mando/dejó - a los niños - correr-INF No FLEX

c. A Maria fez/mandou/deixou correr os miúdos.

María - hizo/mando/dejó - correr-INF NO FLEX - a los niños

d. * A Maria fez/mandou/deixou correrem os mitidos.

María - hizo/mando/dejó - correr-INF FLEX - los niños

(17) a. A Maria fez/mandouldeixou eles correrem.

María - hizo/mando/dejó - ellos - correr-INF FLEX

b. A Maria fê-los/mandou-osídeixou-os correr.

María - hizo/mando/dejo - los - correr-INI: NO ILLEX

c. *A Maria fề-los/mandou-os/deixou-os correrem.

Maria - hizo/mando/dejó - los - correr-INF FLEX

(18) a. A Maria fez/mandou/deixou os miúdos lerem esse livro.

María - hizo/mando/dejó - los niños - leet-INF FLEX - ese libro

b. A Maria fez/mandou/deixou os minidos ler esse livro.

Maria - hizo/mando/dejó - a los niños - leer-INF No FLEX - ese libro

c. A Maria fez/mandou/deixou ler esse livro aos miüdos.

María - hizo/mando/dejó - leer-INF NO FLEX - ese libro - a los niños

(19) a. A Maria fezimandou/deixou eles lerem esse livro.

María - hizo/mando/dejó - ellos - leer-INF FLEX - ese libro

5 El interesante paralclismo sintáctico-semántico entre construcciones causativas y construcciones perceptivas sucede también en otras lenguas románicas, como español, francés c italiano. 
b. A Maria fê-los/mandou-os/deixou-os ler esse livro.

María - hizo/mando/dejó - los - leer-INF NO FLEX - ese libro

c. A Marta fez-lhes/mandou-lhes/deixou-lhes ler esse livro.

María - hizo/mando/dejó - les - leer-INF NO FLEX - ese libro

El verbo causativo puede ser inmediatamente seguido bien por el sujeto lógico del infinitivo, como en $(16 \mathrm{a}, \mathrm{b})$ y $(18 \mathrm{a}, \mathrm{b})$, bien por el infinitivo, como en (16c) y (18c). Pero, cn el primer caso, hay una diferencia entre el infinitivo flexionado $(16 \mathrm{a}, 18 \mathrm{a})^{6}$ y el infinitivo no flexionado $(16 \mathrm{~b}, 18 \mathrm{~b})$. Por el contrario, cuando el verbo subordinado se presenta inmediatamente después del verbo principal, aparece siempre el infinitivo no flexionado, como está ilustrado por la agramaticalidad de (16d). Paso a designar cstas tres estructuras sintácticas como construcciones VSV (16a, 18a), VOV (16b, 18b) y VV (16c, 18c). Además de esta variación del orden de palabras, existe otra que también se refiere al sujeto lógico del infinitivo: es la variación de marcación de caso, envolviendo tres posibilidades?. La no alteración de marcación de caso da origen a la construcción VSV (16a y 18a; con pronombre en nominativo en 17 a y 19a), mientras que VOV (16b, 17b, 18b, 19b) resulta de su codificación como acusativo, u objeto directo del verbo principal. La agramaticalidad de (17c) muestra que el infinitivo flexionado no puede aparecer con acusativo. La construcción VV codifica al sujeto lógico del infinitivo como acusativo (u objeto directo del predicado complejo) cuando la cláusula subordinada es de 1-participante o intransitiva (16c, 17b) , y como dativo (u objeto indirecto del predicado complejo) cuando la cláusula subordinada es de 2-participantes o transitiva $(18 \mathrm{c}, 19 \mathrm{c})$.

VSV, VOV y VV representan tres estadios diferentes en un contimuum de independencia/ integración del evento complemento en relación al evento principal (Givón, 1980): de mayor independencia del evento complemento y monor integración de eventos en VSV a menor independencia del evento complemento y mayor integración de eventos en VV. VSV y VOV son así estructuras bi-oracionales, mientras que VV es una estructura mono-oracional".

La construcción VV es productiva en portugués europeo, sin embargo se tiende a preferir con infinitivo transitivo VOV. La construcción VSV es poco frecuente en portugués europeo. En el portugués de Brasil se encuentran tendencias opuestas: poca productividad de VV (construcción muy rara con infinitivo transitivo) y mayor productividad de VSV.

6 El infinitivo flexionado del portugués manifiesta concordancia en persona y número con el sujeto. Las formas del infinitivo flexionado se construyen a partir de la forma del infinitivo no flexionado más los siguientes morfemas sufijales en concordancia con el sujeto: $-\varphi(1 \mathrm{sg}),-(e) . s(2 \mathrm{sg}),-\emptyset(3 \mathrm{sg}),-m o s(1 \mathrm{pl}),-\operatorname{des}(2 \mathrm{pl}),-m(3 \mathrm{pl})$. El infinitivo flexionado es un fenómeno antiguo y muy típico do la lengua portuguesa, y se manifiesta siempre que el infinitivo se construye con sujeto explicito.

7 La marcación de caso del sujeto lógico del infinitivo es más visible cuando es un pronombre de tercera persona (clítico o no clítico), como en los ejemplos (17) y (19). Las formas de tercera persona del nominativo son ele (masculino), ela (femenino), eles (plural y masculino) y elas (plural y femenino). Las formas nominativas del sistema pronominal portugués son no cliticizables. Los clíticos acusativos de tercera persona son o (masculino), a (femenino), os (plural y masculino) y as (plural y femenino), y adcmás las variantes lo/a/los/las y no/naínos/nas. Los clíticos dativo de tercera persona son the (masculino a femenino) y lhes (plural).

8 De notar que $(17 \mathrm{~b})$ es ambiguo entre VOV y VV. Esto sucede cuandv el verbo subordinado tiene un único argumcnto y el sujeto lógico del infinitivo es cliticizado.

9 Por ser suficientemente conocidas en la literatura, no vamos a describir en pormenor las propiedades estructurales de las tres construcciones (un análisis detallado tanto de las construcciones causativas como de las construcciones perceptivas puede encontrarse en Soares da Silva, en prensa 3). 
VSV, VOV y VV envuelven diferentes atribuciones de destacabilidad focal dentro de la escena complcmonto; en términos de la Gramática Cognitiva de Langacker (1987, 1991, 1999), imposiciones de diferentes perfiles en una misma base (y diferentes organizaciones figurabase y trayector/londmark). Un evento que es causado (en el caso de los verbos de percepción, percibido) constituye la base conceptual común. Más específicamente, las construcciones causativas pcrfilan, tal como Langacker (1991:408) indica, el input de energía o la fuerca instigadora (codificada en el sujeto principal o causante) responsable por la ocurrencia del evento complemento (mientras que las construcciones perceptivas perfilan cl contacto perceptual establecido con el evento complemento por cl sujeto principal o percebidor). Siguiendo a Achard $(1996,1998)$ y nuestro estudio en Soares da Silva (en prensa 3), cl factor determinante de la elección de una construcción particular ticne que ver esencialmente con cuál es la entidad del evento complemento que se ve como inicialmente destacable (la meta inicial de la fuerza o del contacto) -o el evento como un todo, o el participante principal en ese evento (el sujeto lógico del infinitivo) ${ }^{10}$. Es la capacidad de que el sujeto lógico del infinitivo sea considerado como fuente de energia del evento complcmento la que motiva VOV (y VSV) y es la incapacidad de que el mismo participante sea considerado como tal la que determina VV.

Veamos la interpretación específica (o imagen mental) que cada construcción impone en la base común (véase Soares da Silva, en prensa 3, para un análisis más desarrollado). Usando los diagramas de la Gramática Cognitiva, las Figuras 2, 3 y 4 rcpresentan esas tres imágenes específicas. La construcción portuguesa VSV, en la Figura 2, toma todo el evento complemento como meta de la fuerza ejercida (o del contacto establecido) por el sujeto principal; es decir, como landmark del verbo principal. Retomando a los ejemplos (16a) y (18a), el sujeto Maria elabora el irayector del verbo principal y todo el evento complemento os miúdos brincarem en (16a) y os miúdos lerem o livro en (18a) elabora el landmark del mismo verbo. A nivel de la cláusula principal, la destacabilidad focal se atribuye al evento complemento como un todo, y no a sus participantes individuales. Es así como el evento subordinado y su sujeto preservan su autonomía. VSV perfila pues una relación indirecta cntre dos eventos con interacción entre dos trayectores (dos fuentes de energía).

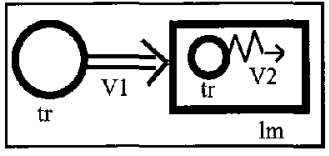

Figura 2. VSV

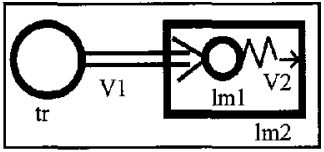

Figura 3. VOV

10 Existen factores más específicos: entre otros, las propiedades semánticas de los verbos principal y subordinado y de sus sujetos; el grado de gramaticalización y de lexicalización (ambas favorables a la construcción VV); y factores discursivos o incluso sintácticos. Por ejemplo. la lexicalización sucede con frecuencia con verbos de perccpción física o mental, como en portugués ver, entender, supor (donde fazer/deixar ver, enterder, supor) y en español hacer ver/creer/pensar/saber (en sentido literal y figurado) y con verbos básicos de movimiento como cair, passar, andar, corro, wi; entrar, parar (deixar cai, passar, andar, correr, en sentido literal y figurado; mandar vir; entrar, parar). Destacable es además un factor particular que imponc restricciones a VV: con infinitivo transitivo y sujcto del infinitivo no pronominalizado, cuanto mayor sea la valencia del infinitivo (o más extensos sean sus complementos), menos fácil scrá VV. 
VOV, en la Figura 3, construye el participante principal del evento subordinado como meta específica de la fuerza o del contacto, o landmark primario (objeto o tema) del verbo principal, pero al mismo tiempo lo reconoce como fuente de energía válida del evento complemento, el cual se toma como landmark secundario del mismo verbo principal, Retomando (16b) y (18b), cl sujeto lógico del infinitivo os miúdos elabora el landmark primario del verbo principal y el evento complomento como un todo os mizidos correr on (16b) e os mizidos ler o livro en (18b) funciona como landmark secundario del mismo verbo principal. VOV perfila, así, una relación también indirecta entre los dos eventos, pero con una interacción más directa entre sus trayectores. El sujeto principal interactúa directamente con el objeto elevado, cl cual se toma como punto de referencia. Es decir, al evento complemento se tiene acceso a través de su trayector o participante principal, y es en la calidad de punto de referencia donde éste ültimo se toma como objeto del verbo principal".

Siguiendo la descripción de Achard (1996:327), la construcción VV, en la Figura 4, envuelve dos atribuciones consccutivas de destacabilidad focal secundaria (o estatuto de landmark). En primer lugar, el evento complemento como un todo se toma como landmark del verbo principal. Sc trata de una operación parecida a la que aparece en VSV, pero con una diferencia esencial: en VSV el sujcto del infinitivo mantiene su destacabilidad inicial, mientras que en esta primera etapa de VV pierde la destacabilidad inicial. Más precisamente, es la actividad o proceso expreso por el infinitivo $\left(V_{2}\right)$ que constituye el landmark de la cláusula principal (o la zona activa del landmark). De aquí resulta precisamente la formación de un predicado complejo $\left(\mathrm{V}_{1+2}\right)$ y, consecuentemente, la perfilación de un único evento. En segundo lugar, el sujeto del infinitivo gana destacabilidad focal, readquierc su destacabilidad intrínseca y pasa a ser perfilado como landmark de este verbo complejo - landmark primario con infinitivo intransitivo (es ésta la imagen que la Figura 4 representa) y landmark secundario con infinitivo transitivo. Retomando a $(16 \mathrm{c})$ y $(18 \mathrm{c})$, durante la primcra operación cognitiva, el infinitivo correr en (16c) y el infinitivo ler en (18c) elaboran el landmark del verbo principal, y así se forma el verbo complejo. Durante la scgunda operación cognitiva, el nominal os miúdos elabora en (16c) el landmark (objeto directo) y en (18c) el landmark secundario (objeto indirecto) de ese verbo complejo. VV construye, así, el participante principal del evento complemento como argumento interno (tema/expcrimentador) de un único verbo complejo y perfila una única actividad o proceso con un único trayector ejerciendo control sobrc todo el evento.

11 Al contrario de las verdaderas consirucciones de clevación, aquí, en VOV, el sujeto principal interacciona directamente con el objeto elevido y se toma ćste como punto de referencia no sólo por ser el trayector del evento en causi, sino también por ser la meta específica o landmark de la fuerza ejercida por el sujeto principal. Esa intcracción directa es más evidente con los verbos causativos (que con los verbos perceptivos) e incluso más con fazer y mandar que con deixar. Consecucntemente, el verbo principal impone restricciones en el objcto elevado. En términos de la noción de transparencia de Langacker (el hecho de que uany element that can occur in the appropriate position in the subordinate clause can likewise occur in 'raised' position in the main clause", Langacker, 1995:40), la construcción VOV exhibe transparencia mínima en relación a la selección de su objeto. 


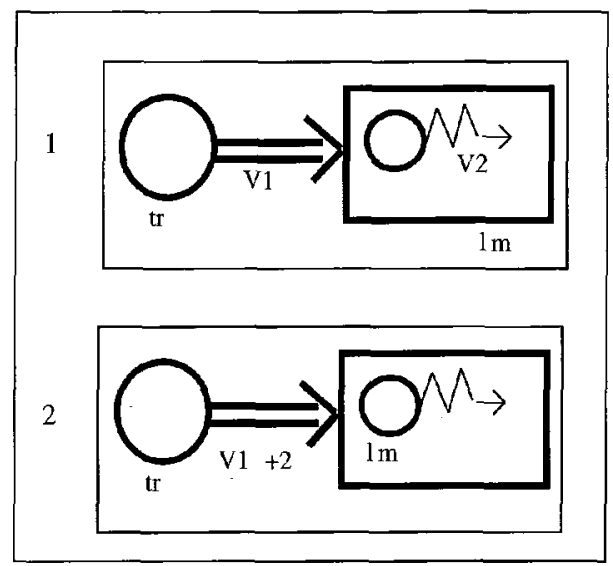

Figura 4. VV

Desde VSV hasta VV aparece un cambio que puede describirse en términos de Langacker (1999: cap. 10) como proceso progresivo de at a en el segundo; cfr. Fauconnier y Turner, 1996, para el francés); sin embargo, la coherencia de VV continúa.

VOV es, en portugués, exclusiva de los verbos en análisis. Además, presenta varias similitudes con las llamadas construcciones de control (control de objeto). Y VSV presenta un comportamiento idéntico al de las variadas cláusulas de complemento infinitivo flexionado existentes en portugués ${ }^{2}$.

Otra construeción de complemento de infinitivo donde participan otros verbos causativos es VOaV, ejemplificada en (12) y que aquí repetimos.

(12) a. A Maria forçalobriga/leva as crianças a sairem.

María - fuerza/obliga/lleva - los niños - a salir-INF FI.EX

b. A Maria força'obrigalleva as crianças a sair.

Maria fuerza/obliga/lleva a los niños a salit.

Esta construcción de infinitivo con preposición, del portugués, español y de otras lenguas románicas, simboliza una distancia mayor entre el evento causante y el evento calusado, icónicamente marcada por la preposición $a$, la cual hace explícito el recorrido que progresivamente conducc al causado en dirección a la realización del evento expreso en el infinitivo (Achard 1998:104, 2002, relativamente a la misma construcción del francés con el verbo forcer). VOaV exhibe, asi, similitudes con las construcciones de movimiento causado y ditransitiva: todas comparten el significado de movimiento causado de una en-

12 En portugués, las cláusulas de infinitivo flexionado uparecen como cláusulas sujeto, cláusulas adjunto introducidas por una preposición y cláusulas complemento de diferentes tipos de verbos. La forma VSV es igual a la de cláusulas complemento de infinitivo flexionado de verbos como lamentar, aprovar, censurar, tolerar: por ejemplo, Eu lamento eles terem assinado a deciaração [Lamento - ellos - haber-inf flex firmado - la declaración]. 
tidad en dirección a una meta, codificada en la preposición a. Mientras VOaV expresa un esquema causativo progresivo, las construcciones causativas infinitivas anteriores expresan un esquema causativo incoativo. Además, VOaV está más próxima de VOV que de VSV, porque cl sujeto del infinitivo con preposición tiene que marcarse en acusativo, como objeto del verbo causativo; por eso mismo, VOaV perfila, tal como VOV, una interacción más directa entre causante y causado. La variación entre infinitivo flexionado (12a) e infinitivo no flexionado (12b) aparece orientada por los mismos factores que están en la base de la variación entre VSV y VOV.

\section{COMPATIBILIDAD ENTRE CONSTRUCCIONES Y VERBOS}

Combinando los significados de las construcciones causativas con complemento infinitivo con los significados de los verbos causativos que en ellas participan, sería de esperar que fazer seleccionase la construcción VV y deixar la construcción VOV (y VSV). Sería también de esperar que la construcción mono-oracional $V V$ fuese rescrvada para codificar la causación física directa y las construcciones bi-oracionales VOV y VSV se reservasen para codificar la causación inductiva, propia de la interacción humana. Esto, además, no sucede, como ya se ha podido comprobar en los ejemplos (16)-(19). Y el hecho más sorprendente es que el verbo portugués fazer, al contrario de todos sus equivalentes románicos, toma también VOV (y VSV) ${ }^{13}$. De hecho, hay una mayor flexibilidad en la combinación de verbos y construcciones causativas en portugués que en las otras lenguas románicas ${ }^{14}$.

Muy sumariamente, veamos algunos ejemplos más de la interacción entre verbos y construcciones causativas en portugués (véase Soares da Silva, 2004, en prensa 3, para un análisis más desarrollado).

La distribución de VSV-VOV y VV con fazer y deixar está determinada, en parte, por cl factor de la actividad de lo causado, referido en la sección anterior. Es así como causados no-animados tienden a usarse en la construcción VV, como en (20) y (21).

(20) a. A Maria deixou cair o livro. (VV)

b. ??A Maria deixou o liwro cair. (VOV)

'María dejó caer el libro.'

(21) a. A Maria fez cair o livro. (VV)

b. ??A Maria fez o livro cair. (VOV)

'María hizo caer el libro.'

13 La alternancia VOVNV no es posible con francés faire, italiano fare y (generalmente, por lo menos) cspañol haceer, los cuales aparecen tan sólo en la construcción VV (cfr. Rocgiest, 1983). En relación al español, Treviño (1992:310) reficrc la posibilidad de que hacer pueda ser seguido de sujeto del infinitivo y, así, admitir, aunque raramente, la constucción VOV, como en Juan hizo a Pedro abrir la puerta. La nisma posibilidad la señala Comrie (1976:303) con el ejemplo Hice a las criadas construir el edificio, en oposición a Hice construir el edificio a las criadas. Véase también Zubizarreta (1985). En casos normales, hacer-VOV no es posible: *Iicieron los soldados destmir la ciudad, sin embargo sí Hicieron destruir la ciudad a/por los soldados (Bordelois, 1988:57-58).

14 El recurso a la construcción VOV es frecuente en portugués, no existiendo restricciones ni sobre el verbo principal ni sobre el número de argumentos del infinitivo. Véase $A$ Maria fez/mandou/deixou o minido entregar o livm ao professor (VOV), sin cmbargo *A Maria fezimandouddeixou entregar o livro ao professor ao miudo (VV). 
Pero cuando to causado no animado es conccptualizado como fuente de energía del evento expreso en el infinitivo, entonces VOV se selecciona, como en (22)-(23), o incluso VSV, como en (24).

(22) Deixa o leite subir até cimal

'iDeja que la leche hierva!'

(23) Fez a bola (ganhar altura e) passar por cima do guarda-redes.

'Hizo que cl balón (ganase altura y) pasase por encima del portero.'

(24) Muitos milhões de dólares já foram sacrificados a esta ambição de fazer os carros andarem a electricidade.

'Muchos millones de dólares ya se han sacrificado por esta ambición de hacer que los coches anden con electricidad.'

Causados humanos, porque son prototípicamente activos, serían entonces más compatibles con las construcciones VOV y VSV. Sin embargo, las cosas se complican ahora aún más, porque otros factores pueden ser determinantes. Con efecto, la causación a nivel intencional (e interpersonal) es más compleja que la que aparece a nivel (puramente) físico. Comparemos los ejemplos (a) y (b) de (25)-(26).

(25) a. Bush fez regressar os seus marines ao Iraque. (VV)

b. Bush fez os seus marines regressariem ao Iraque. (VOV/VSV)

'Bush hizo regresar a sus marines a Irak.'

(26) a. Bush deixou regressar os seus marines ao Iraque. (VV)

'Bush dcjó regresar a sus marines a Irak.'

b. Bush deixou os seus marines regressarlem ao Iraque. (VOV/VSV)

'Bush dejó a sus marines regresar a Irak.'

Tanto en (25a) como en (26a), el regreso de los marines a Irak se ve como directamente inducido por Bush, por lo que éste hizo algo en (25a) o no hizo nada en (26a) contra la tendencia intrínseca de los marines. Bush se muestra como aquél que tiene entera responsabilidad por ese regreso, por lo que la actividad de los marines no es perfilada. De ahí la construcción VV. En (25b) y (26b), Bush es igualmente responsable por el regreso de los marines a Irak, sin embargo ahora cstc regreso sc ve como dependiente también de los marines, del cese de su resistencia y de su capacidad de iniciar ese proceso forzado, en (25b), o de su voluntad inicial en querer realizar ese proceso (pidiendo autorización), en (26b). $\mathrm{O}$ sea, el papel activo de lo causado es ahora perfilado y su intervención activa en el evento inducido/autorizado es especificada, lo que requiere VOV-VSV.

El sentido más coercivo de fazer es factor suficiente para la selección de VOV (o VSV), como ilustrado en (27). Por su parte, el sentido normativo de deixar, dado que presume autoridad de lo causante y un pedido implícito de permiso por parte de lo causado, tiende a ser más compatible con VOV, como en (28). Y el sentido de 'no intervenir' del mismo verbo, como perfila una actitud pasiva de lo causante, tenderá a scr también más compatible con la construcción bi-oracional, como en (29). Sin embargo con causados no-animados ya es más probable encontrar este sentido 'pasivo' en VV, como deixar caducar o prazo (??deixar o prazo caducar) ['dejar caducar el plazo'] o deixar crescer o cabelo (?deixar o cabelo crescer) ['dejar crecer el pelo']. Todo esto, además, son simples tendencias, que pueden contrariarse con factores pragmáticos u otros. 
(27) a. A mãe fez o(s) fitho(s) estudar(em) durante duas horas. (VOV/VSV)

b. ??A mãe fez estudar of filho durante duas horas. (VV)

'La madre hizo que el/los hijo(s) estudiase(n) durante dos horas.'

(28) a O pai não deixou a Maria fumar. (VOV)

'El padre no dejó a María fumar.'

b. ?O pai não deixou fumar a Maria. (VV)

'El padre no dejó fumar a María.'

(29) a. A Maria é irresponsável: deixa o filho fazer o que ele quer. (VOV

'María es irresponsable: deja al hijo hacer lo que quiere.'

b. ?A Maria é irresponsável: deixu fazer ao filho o que ele quer. (VV)

'María es irresponsable: le deja hacer lo que quiere.'

En lo que se refiere a mandar, las características de orden directa y causación directa e intencional, pero no implicativa y no inmediata, explican su tendencia para la construcción bi-oracional VOV, como aparece ilustrado en (30). Pero la posibilidad de no explicitación del destinatario abre camino a la construcción VV (real o aparente).

(30 a. Mandei o rapaz ir embora. (VOV)

'Mande al chaval irse.'

b. ??Mandei ir embora o rapaz. (VV)

'Mandé irse al chaval.'

\section{LA CONSTRUCCIÓN CON COMPLEMENTO FINTTO Y OTRAS CONSTRUCCIONES CAUSATIVAS}

Una construcción causativa alternativa a las anteriores es la construcción de complemento finito, introducida por el complementador que - precedido de la preposición com en el caso del verbo fazer y de la preposición a con los verbos que entran en la construcción de infinitivo preposicionado- y con verbo en subjuntivo, ejemplificada en (13) y que aquí repetimos.

(13) a. A Maria faz com que/manda'deixa que as crianças saiam. Maria hace/manda/deja que los niños salgan.

b. A Maria força/obriga/leva a que as crianças saiam. Maria fuerza/obligalleva a que los niños salgan.

Esta construcción, existente en las lenguas románicas pero menos frecuente que la construcción infinitiva, codifica la mayor independencia posible del evento causado, es dccir un grado encima de la construcción portuguesa VSV - compárese (13a) con (11a) A Maria faz/manda/deixa as crianças sairem (Maria - hace/manda/deja - los niños - salir-INF FLEX). La construcción con complemento finito marca, icónicamente, una distancia conceptual entre los dos eventos y expresa una interpretación holística, abstracta y atemporal del cvento causado (véase la caracterización de Langacker, 1991:439-449, de los complementadores). En VSV, el evento causado se construye también holísticamente, pero no abstracta ni atcmporalmente.

¿Y cuál es la razón del subjuntivo? Siguiendo la explicación de Achard (1998: cap. 6), el subjuntivo indica que la cláusula complemento se considera, no en relación a la realidad, sino en relación a un espacio mental más circunscrito, solamente relevante al sujeto princi- 
pal. Los verbos causativos son exclusivamente compatibles con el subjuntivo, en perfecta sintonia con el hecho de que su complemento no es parte de la realidad sino resultado del input de energía del sujeto principal. ${ }^{15}$

La construcción con complemento finito asume significados más especificos consonante al verbo causativo que en ella participa. Así, la construcción fazer com que/hacer que vuclvc más explícita la relación causal y, con causante humano, implica que éste actúe intencional y premediladamente. La construcción deixar que/dejar que cxpresa también un acto deliberado y premeditado de lo causante, por lo que cuando éste toma una actitud pasiva de no interferencia esa pasividad es siempre reflexionada (y en este sentido activa). Y la construcción mandar que no implica que el orden sea dado/recibido directamente. Complementalmente, tiene la función pragmática de suavizar la exigcncia del ordenante, por lo que esta construcción es la que se utiliza en un acto normal de dar una orden a alguien - cfr. en portugués Mando (Ordeno-te) que saias! 'Mando (Te ordeno) que salgas' vs. ??Mando-te ( Ordeno-te) sair! 'Te mando (Te ordeno) salir'.

La construcción causativo-reflexiva, ejemplificada con anterioridad en (14), es admitida por los causativos canónicos fazer/hacer (sólo con infinitivo transitivo), deixar/dejar (con infinitivo intransitivo ergativo y con infinitivo transitivo), pero no por mandar, y por algunos de los causativos con infinitivo con preposición, como obrigar/obligar. La corrcferencialidad ocurre entre causante y causado con deixar/dejar e infinitivo intransitivo y con obrigariobligar; y entre causante y afectado con fazer/hacer y deixar/dejar e infinitivo transitivo. La construcción causativo-reflexiva evidencia un interesante conflicto de fuerzas interior al participante principal y por éste controlado (véase cl cstudio de García-Miguel, 2003, para el español).

Se añade tan sólo un breve apunte sobre la construcción causativa predicativa, ejemplificada antes en (15) A Maria/Isso fez-me/pôs-me/tornou-me/deixou-me feliz. / Maria/Eso me hizo'me puso/me volvióme dejó feliz. Esta construcción perfila el resultado de la causación - causar la adquisición de una cualidad o la entrada en un estado-, por lo que puede designarse como causativo-resultativa. EI argumento predicativo del objeto directo (causado) toma la forma de un adjetivo, participio o sintagma preposicional y expresa el estado de lo causado como resultado del evento causante. Comparando los cuatro verbos que en ella participan, fazer/hacer y sobretodo tornar/volver presuponen que el estado en el cual lo causado ingresa cs permanente, mientras que pôr/poner presupone que ese estado es transitorio; y deixar/dejar focaliza no el cambio y por tanto no el ingreso del objeto en cl nucvo estado, sino el punto de llegada o el resultado de ese cambio. Es así como algunos usos (pero no otros, como 4b, antes) de deixar y predicativo del objeto directo - lo mismo sucede con dejar; véase el estudio de Roca Pons (1955) sobre la perifrasis dejar + participio, y también Aranda Ortiz (1990:181-186) - se aproximan de una causación del tipo 'hacer', pero perspectivada como resultado, ya que la alteración está seguida por la partida de lo causante.

\section{CONCLUSIÓN}

Contrariamente a una larga tradición formalista (generativista), la complejicad de las construcciones causativas analíticas del portugués y español se vuelve explicable a la luz de su contenido semántico y de sus motivaciones conceptuales - resultado que corrobora las conclusiones de Achard (1996, 1998) para cl francés. Importantes constructos de modelos 
gramaticales y semánticos de la Lingüística Cognitiva se han revelado decisivos para este resultado. El análisis de los verbos causativos muestra quc conceptualizamos (mctafóricamente) las causas como fuerzas y la causación en un escenario de interacción de fuerzas donde una entidad posee una tendencia natural hacia el movimiento o hacia el reposo y la manifestará a menos que sea vencida por otra entidad más fuerte. 'Hacer + infinitivo' y 'dejar + infinitivo' constituyen los dos padrones básicos de lexicalización y gramaticalización de la causación, siendo el primero prototípico-sugiriendo así que conceptualizamos 'causar' en términos de 'hacer' y los efectos como objetos hechos- y el segundo la contrapartida negativa del primero. El análisis de las construcciones causativas de complemento infinitivo y finito evidencia un continuum de integración/independencia de los eventos causado y causante y de autonomía del participante causado y control del participante causante.

Las construcciones causativas analíticas del portugués presentan un continuum más elaborado de integración de cventos y una imaginaria (mental) más rica acerca del evento complemento, que el español y otras lenguas románicas. $Y$ los diferentes verbos causativos del portugués exhiben mayor flexibilidad en la estructuración de su complemento y en la integración en cada una de las construcciones. Consecuentemente, la autonomía del significado de las construcciones aumenta y la intcracción entre significado del verbo y significado de la construcción se vuelve más flexible y más dinámica.

\section{REFERENCIAS BIBLIOGRÁTICAS}

Achard, Michel (1996): «Two causation/perception constructions in French», Cognitive Linguistics 7, págs. 315-357.

Achard, Michel (1998); Representation of Cognitive Structures. Syntax and Semantics of French Sentential Complements. Berlin, Mouton de Gruyter.

Achard, Michel (2002): «Causation, constructions, and language ecology: An example from French». En Shibatani, M. (ed.), págs. 127-155.

Aranda Ortiz, Antonio (1990): La Expresión de la Catsatividad en Español Actual. Zaragoza, Libros Pórtico.

Bernárdez, Enrique (2001): «Cultural determination of cause-effect. On a possible folk model of causation», Circle of Linguistics Applied to Communication 6: http://www.uem.es/info/circulo/ no6rbernardez.htm

Bordelois, Ivonne (1988): «Causatives: From lexicon to syntax», Natural Language and Linguistic Theory 6, págs. 291-352.

Cano Aguilar, Rafael (1981): Estructuras Sintácticas Iransitivas en el Español Actual. Madrid, Gredos.

Comrie, Bernard (1976): "The syntax of causative constructions: cross-language similarities and divergences», En Shibatani, M. (ed.): Syntax and Semantics 6: The Grammar of Causative Consiructions. New York, Academic Press, págs. 261-312.

Fauconnier, Gilles y Mark Turner (1996); «Blending as a central process of grammar», En Goldberg. A. (ed.): Conceptual Structure, Discourse and Language. Stanford, CSLI Publications, págs. 113-130.

García-Miguel, José M. (2003): «Integración semántica en las consirucciones causativas reflexivas en españoly. En Delbecque, N. (ed.): Aproximaciones Cognoscitivo-Funcionales al Español (Foro Hispánico. Revista Hispánica de Flandes y Holanda, 23). Amsterdam, Rodopi.

Givon, Talmy (1980): «The binding hierarchy and the typology of complements», Studies in Language 4, pägs. $333-377$.

Goldberg, Adele E. (1995): Constructions. A Construction Grammar Approach to Argument Structure. Chicago, The University of Chicago Press. 
Jackendoff, Ray (1990): Semantic Structures. Cambridge, Mass., The MIT Press.

Kemmer, Suzanne y Arie Verhagen (1994): «The grammar of causatives and the conceptual structurc of events», Cognilive I,inguistics 5, págs. 115-156.

Langacker, Ronald W. (1987): Foundations of Cognitive Grammar, Vol. 1: Theoretical Prerequisites. Stanford, Stanford University Press.

Langacker, Ronald W. (1991): Foundations of Cognitive Grammor, Vol. 2: Descriptive Application. Stanford, Stanford University Press.

Langacker, Ronald W. (1995): «Raising and transparency», Language 71, págs. 1-62.

Langacker, Ronald W. (1999): Grammar and Conceptualization. Berlin/New York, Mouton de Gruyter.

Roca Pons, José (1955): «Dejar + Participio», Revista de Filología Española 39, págs. 151-185.

Roegiest, Eugeen (1983): «Degrés de fusion dans la construction factitive des langues romancs». En Roegiest, F. y L. Tasmowsky (eds.): Verbe et Phrase dans les Langues Romanes. Mélanges offerts à Louis Morin, (Romanica Gandensia XX), Gent, págs. 271-288.

Shibatani, Masayoshi y Prashant Pardeshi (2002): «The eausative continuum». En Shibatani, M. (ed.), págs. 85-126.

Shibatani, Masayoshi (ed.) (2002): The Grammar of Causation and Interpersonal Manipulation. Amsterdam, John Benjamins.

Soares da Silva, Augusto (1999): A Semântica de DEIXAR: Uma Contribuiçâo para a Abordagem Cognitiva em Semântica Lexical. Lisboa, Fundaçâo Calouste Gulbenkian y Fundação para a Ciência e a Tecnologia.

Soares da Silva, Augusto (2000): «The 'letting' causation: Evidence from Portuguese». Paper presented at the Winter Symposium Structures of Causal Meaning. Center for Scmiotics, University of Aarhus, 27-29 January 2000. www.hum.au. dk/semiotics

Soares da Silva, Augusto (2003a): uImage schemas and calegory coherence: The case of the Portuguese verb deixar», En Cuyckens, H., R. Dirven y J. R. Taylor (eds.): Cognitive Approaches to Lexical Semantics, Berlin/New York, Mouton de Gruyter, págs. 281-322.

Soares da Silva, Augusto (2003b): «La structure sémantique de 'laisser' dans les langues romanes». En Sánchez Miret, F. (ed.): Actas del XXIII Congreso Internacional de Linguiistica y Filologia Románica, vol. III. Tübingen, Max Niemeyer Verlag, págs. 441-456

Soares da Silva, Augusto (2004): «Cultural determinations of causation». En: Soares da Silva, A., A. Torres y M. Gonçalves (eds.): Linguagem, Cultura e Cognição: Estudos de Linguística Cognitiva, vol. I. Coimbra, Almedina, págs. 575-606.

Soares da Silva, Augusto (en prensa 1): «Verbs of letting: Same cognitive and historical aspects». En Delbecque, N. y B. Cornillie (eds.): Causation and Motion. Amsterdam, John Benjamins.

Soares da Silva, Augusto (en prensa 2): ('Leave vs. keep things as they are' from a force dynamic perspective), En Graumann, A., P. Holz y M. Plümacher (eds.): Towards a Dynamic Theory of Lamguage. Studies in honour of Wolfgang Wildgen. Bochum, Universitätsverlag Dr. N. Brockmann.

Soares da Silva, Augusto (en prensa 3): «Imagery in Portuguese causation/perception constructions». En Lewandowska-Tomaszczyk, B. y A. Kwiatkowska (eds.): Imagery in Language. In honour of Professor Ronald W. Langacker. Frankfurt/Main, Pcter Lang.

Talmy, Leonard (1988): «Force dynamics in language and cognition», Cognitive Science 12, págs. 49-100.

Talmy, Leonard (2000): «Force dynamics in language and cognilion». En Talmy, L.: Toward a Cognitive Semantics, vol. I: Concept Structuring Systems. Cambridge, Mass., The MIT Press, págs. 409-470

Treviño, Esthela (1992): «Subjects in Spanish causative constructions». En Hirschbühler, P. y K. Koemer (eds.): Romance Languages and Modern Linguistic Theory. Amsterdam, John Benjamins, págs. 309-324.

Zubizarrcta, M. Luisa (1985): «The relation between morphophonology and morphosyntax: the case of Romance causatives», Linguistic Inquiry 16-2, págs. 247-289. 